\section{KẾT LUẬN}

Các kết quả nghiên cứu của chúng tôi thu được cho thấy: Phương pháp phẫu thuật ít xâm lấn có nội soi hỗ trợ, tim đập để đóng thông liên nhĩ qua đường mở ngực phải tại Bệnh viện Hữu nghị Việt Đức đảm bảo an toàn, hiệu quả và kết quả tốt.

\section{TÀI LIẸU THAM KHẢO}

1. Liu $\mathbf{Y}$, Chen S, Zühlke $\mathbf{L}$, et al. Global birth prevalence of congenital heart defects 1970-2017: updated systematic review and meta-analysis of 260 studies. Int J Epidemiol. 2019;48(2):455-463. doi:10.1093/ije/dyz009

2. Nathan M. Do the benefits outweigh the risks? A review of the history of atrial septal defect repairs and device closures. :2.

3. Baumgartner $H$, De Backer J, Babu-Narayan SV, et al. 2020 ESC Guidelines for the management of adult congenital heart disease. Eur Heart J. 2021;42(6):563-645. doi:10.1093/ eurheartj/ehaa554

4. Ooi YK, Kelleman $M$, Ehrlich $A$, et al. Transcatheter Versus Surgical Closure of Atrial Septal Defects in Children. JACC Cardiovasc Interv.
2016;9(1):79-86. doi:10.1016/j.jcin.2015.09.028

5. Dang Q-H, Le $\mathbf{N}-\mathbf{T}$, Nguyen $\mathbf{C}-\mathbf{H}$, et al. Totally Endoscopic Cardiac Surgery for Atrial Septal Defect Repair on Beating Heart Without Robotic Assistance in 25 Patients. Innov Phila Pa. 2017;12(6):446-452. doi:10.1097/IMI.0000000000000436

6. Kodaira M, Kawamura A, Okamoto $K$, et al. Comparison of Clinical Outcomes After Transcatheter vs. Minimally Invasive Cardiac Surgery Closure for Atrial Septal Defect. Circ J. 2017;81(4):543-551. doi:10.1253/circj.CJ-16-0904

7. Mylonas KS, Ziogas IA, Evangeliou A, et al. Minimally Invasive Surgery vs Device Closure for Atrial Septal Defects: A Systematic Review and Meta-analysis. Pediatr Cardiol. 2020;41(5):853861. doi:10.1007/s00246-020-02341-y

8. Ma Z-S, Dong M-F, Yin Q-Y, Feng Z-Y, Wang L-X. Totally thoracoscopic closure for atrial septal defect on perfused beating hearts. Eur J Cardiothorac Surg. 2012;41(6):1316-1319. doi:10.1093/ejcts/ezr193

9. Nassif $M$, van der Kley $F$, Abdelghani $M$, et al. Predictors of residual tricuspid regurgitation after percutaneous closure of atrial septal defect. Eur Heart J - Cardiovasc Imaging. 2019;20(2):225232. doi: $10.1093 /$ ehjci/jey080

\title{
TÁC ĐộNG CỦA ĐIỀU TRỊ PHỤC HÌNH RĂNG LÊN CHẤT LƯợNG CUộC SỐNG CỦA BỆNH NHÂN ĐẾN ĐIỀU TRI TẠI BỆNH VIỆN RĂNG HÀM MẶT THÀNH PHỐ Hồ CHÍ MINH
}

\section{TÓM TẮT}

Mục tiêu: Đánh giá tác động của tình trạng mất răng lển chất lượng cuộc sống. Đánh giá hiệu quả của điều trị phục hình răng lên chất lượng cuộc sống của bênh nhân sau 1 tháng điều trị. Đối tượng và phương pháp nghiên cứu: Nghiên cứu cắt ngang mô tả theo dõi trước sau, thực hiên trên đối tượng mất răng có chỉ định điêu trị phục hình cố định hoặc phục hình tháo lắp bán phần tại khoa Phục hình bệnh viển Răng Hàm Mặt Tp. Hồ Chí Minh trong thời gian từ tháng 8/2020 đến tháng 2/2021. Bộ câu hỏi OHIP-14 được dùng để khảo sát chất lượng cuộc sống liên quan đến tình trang sức khoẻ răng miệng của bênh nhân trước điêu trị và 1 tháng sau điêu trị phục hình răng qua phỏng vấn trực tiếp bệnh nhân. Kểt quả: Mẫu nghiên cứu gồm 79 đối tượng (52 nữ, 27 nam) có độ tuổi trung bình là 47,5 $\pm 11,9$ (24 - 74 tuổi). Có $5 \dot{6}$ người $(70,9 \%)$ đã từng mang hàm giả, thời gian mất

*Bệnh viện Răng Hàm Mặt, Tp.HCM

**Đai hoc Y Dước Thành phố Hồ Chí Minh

Chịu trách nhiệm chính: Trân Thu Trang

Email: mimitran0206@gmail.com

Ngày nhận bài: 22.3.2021

Ngày phản biên khoa học: 13.5.2021

Ngày duyệt bài: 21.5.2021
Trần Thu Trang*, Trần Thu Thủy**

răng trung bình là 106,4 tháng, thời gian mang hàm giả trung bình là 88,5 tháng. Điểm trung bình OHIP-14 trước điêu trị là $18,1 \pm 10,8$ và sau phục hình 1 tháng là $9,6 \pm 7,4$. Chất lượng cuộc sống được cải thiện đáng kể sau 1 tháng điêu trị phục hình $(p<0,001)$. Kết luận: Kết quả nghiên cứu cho thấy có sự cải thiên chất lượng cuộc sống sau điều trị phục hình cố định hoặc/và phục hình tháo lắp bán phần 1 tháng.

Ti̛ khóa: chất lượng cuộc sống, OHIP-14, phục hình cố định, phục hình tháo lẳp bán phân.

\section{SUMMARY}

THE IMPACT OF ORAL HEALTH ON QUALTTY OF LIFE IN PARTIALLY EDENTULOUS PATIENTS

BEFORE AND AFTER PROSTHODONTIC REHABILITATIONS OF THE ODONTO-MAXILLO FACIAL HOSPITAL HO CHI MINH CITY

Objectives: Changes in oral health-related quality of life (OHRQoL) for partially edentulous patients treatment at the department of Prosthodontic of the Odonto-Maxillo Facial hospital Ho Chi Minh city according to the OHIP-14. Change in OHRQoL after prosthetic treatments 1 month at the department of Prosthodontic of the Odonto-Maxillo Facial hospital Ho Chi Minh city. Method: Descriptive study, follow-up, performed on 79 patients with teeth loss needing 
treatment for fixed prosthesis or removable partial denture at the department of Prosthodontic of the Odonto-Maxillo Facial hospital Ho Chi Minh city. Change in OHRQoL after prosthetic treatments 1 month according to the OHIP-14 index. Results: The mean age of the study participants was 47.5 years, female accounted for $65.8 \%, 68,4 \%$ of subjects are wearing old dentures. Median OHIP-14 scores were $18,1 \pm 10,8$ and after prosthetic treatments 1 month median OHIP-14 scores were 9,6 $\pm 7,4$. OHRQoL improved significantly after prosthodontics treatment 1 month $(p<0.001)$. Conclusion: The results showed that OHRQoL improved significantly after 1 month of prosthetic treatments.

Key words: quality of life, OHIP-14, fixed prosthesis, removable partial denture.

\section{I. ĐĂT VẤN ĐỀ}

Tổ chức Y Tế Thế Giới (WHO) đinh nghĩa "Sức khỏe là tình trang hoàn toàn khỏe manh về mặt thể chất, tinh thần và xã hội, chứ không chỉ đơn thuần là không bệnh tật" [5]. Vì vậy, đánh giá hiệu quả chăm sóc y tể không còn chỉ dựa vào việc chúng ta sống bao lâu mà quan trọng là chúng ta sống thế nào. Chính điều này làm thay đổi hướng tiếp cận chăm sóc sức khỏe và các khái niệm chất lượng cuộc sống trong đó có chất lương cuôc sống liên quan đến sức khỏe răng miệng (CLCS-SKRM) được ra đời.

Nhiều nghiên cứu thế giới cho thấy mối liên quan giữa chất lượng cuộc sống và sức khỏe răng miêng. Nhiều nghiên cứu nhấn mạnh tình trạng mất răng toàn bộ gây ra những hậu quả về cảm xúc và tâm lý như những rối loạn khác của cơ thể. ớ Việt Nam có vài nghiên cứu về tác động của điều trị phục hình răng tháo lắp toàn bộ lên chất lượng cuộc sống như Nguyên Thanh Hương (2017), Nguyễn Ngọc Đạt (2018) nhưng chưa có nghiên cứu nào nói về tác động của phục hình cầu răng, tháo lắp bán phần lền chất lượng cuộc sống. Nghiên cứu của Swelem và CS (2014) [8], $A m j$ id $N$ và $C S$ (2019) [1], Katerina $Z$ và $C S$ (2020) [2], cho thấy phuc hình cố định và phục hình tháo lắp bán phần giúp cải thiện CLCS.

Vì vậy, chúng tôi tiến hành nghiên cứu "Tác đông của điều trị phục hình răng lên chất lương cuộc sống của bệnh nhân đến điều trị tại bềnh viện Răng Hàm Mặt thành phố Hồ Chí Minh" với mục tiêu: 1) Đánh giá tác động của tình trang mất răng lên chất lượng cuộc sống của bềnh nhân đến điều trị tại khoa Phục hình bệnh viện Răng Hàm Mặt TPHCM theo chỉ số OHIP - 14. 2) Đánh giá hiệu quả của điều trị phục hình răng lên chất lượng cuộc sống của bệnh nhân đến điều trị tại khoa Phục hình bệnh viện Răng Hàm Mặt TPHCM sau 01 tháng điêu trị theo chỉ sô OHIP - 14.

\section{II. ĐỐI TƯƠNG VÀ PHƯƠNG PHÁP NGHIÊN CỨU}

Thiết kế nghiên cứu: Nghiên cứu mô tả theo dõi trước sau.

Đối tượng nghiên cứu: Chọn các đối tượng mất răng cần điều trị phục hình cầu răng hoặc/ và phục hình tháo lắp hàm khung đến điều trị tai khoa Phục hình Bênh viện Răng Hàm Măt thành phố Hồ Chí Minh từ tháng 8/2020 đến tháng 2/2021.

Cỡ mẫu và phương pháp nghiên cúu: Chọn toàn bộ 79 đối tượng thỏa tiêu chí chọn vào và loại ra trong thời gian từ tháng $8 / 2020$ đến tháng 2/2021.

Dữ liệu nghiên cứu được các bác sĩ điều trị ghi nhận qua khám lâm sàng và phỏng vấn trực tiếp bệnh nhân để đánh giá chất lượng cuộc sống của đối tượng qua thang điểm đánh giá OHIP - 14 trước khi phục hình và sau mang phục hình 1 tháng.

Tiêu chuẩn lựa chọn: Đối tượng mất một hoặc nhiều răng trên một hoặc 2 cung hàm cần điều trị phục hình. Đối tượng minh mẩn và đồng ý tham gia nghiên cứu sau khi nghe giải thích rõ về muc đích và yêu cầu của nghiên cứu.

Tiêuu chuẩn loại trừ: Đối tượng không hiểu tiếng Việt, có bệnh toàn thân nặng như ung thư, suy giảm miễn dịch, bệnh thận mãn tính (có chạy thận), hội chứng Down, đau mạn tính hoặc các rối loan tâm thần/thần kinh. Đối tượng không đủ năng lực trả lời câu hỏi nghiên cứu.

Phương pháp đánh giá: Bộ câu hỏi OHIP14 [7] gồm 14 câu hỏi chia làm 7 lính vực: giới han chức năng, đau thưc thể, không thoải mái tầm lí, thiểu năng về thể chất, thiểu năng về tâm lí, thiểu năng về xã hội và tàn tật. Các mức độ trả lời cho mỗi câu hỏi cùng điểm số từng mức như sau: "0": không bao giờ, "1": hiếm khi, "2": thỉnh thoảng, "3": khá thường xuyên, "4": rất thường xuyên. Áp dụng phương pháp đơn giản để tính điểm số OHIP, nghĩa là cộng điểm của các câu trả lời lại với nhau.

Kiểm soát sai lệch chọn lựa và sai lệch thông tin: tuân thủ chọn mẫu thuận tiện tất cả các đối tượng thỏa các tiêu chí chọn vào và loai ra từ tháng 8/2020 đến tháng 2/2021. Các bác sĩ lâm sàng thực hiện phục hình răng được tập huấn định chuẩn để thống nhất phương pháp thực hiên. Những người thu thâp số liệu được tập huấn trước khi phỏng vấn bệnh nhân về bảng câu hỏi chất lượng cuộc sống và khai thác bêenh sử.

Nhầp liệu và xử lý số liệu: Nhập liệu bằng Excel 2010, phân tích bằng SPSS 20.0. Thống kê mô tả dùng tần số và tỉ lệ \%, trung bình, độ lệch chuẩn, giá trị nhỏ nhất, lớn nhất. Các phép kiểm 
thống kê sử dụng là T-test bắt cặp, phép kiểm chính xác Fisher, Kiểm định Wilcoxon, Mann Whitney, Kruskal-Wallis với mức ý nghĩa $p<0,05$.

Đạo đức nghiên cứu: nghiên cứu đã được thông qua Hội đồng Đạo Đức của Đại Học Y Dược Tp.HCM theo quyết định số 451/HĐĐĐĐHYD ngày $21 / 7 / 2020$. Đối tượng tham gia nghiên cứu đều tình nguyện và được thông tin rõ ràng về nghiên cứu và được ký phiếu đồng ý tham gia nghiên cứu.

\section{KẾT QUẢ NGHIÊN CỨU}

Đặc điểm cửa đối tượng nghiên cứu

Bảng 1. Các đặc điểm của mẫu nghiên cứu $(n=79)$

\begin{tabular}{|c|c|c|}
\hline Đặc điếm của mâu & Tân số (n) & Tỉ lệ (\%) \\
\hline Tuối & $\begin{array}{l}\text { TB } \pm \text { DLC: } \\
47,5 \pm 11,9\end{array}$ & $\begin{array}{c}\text { NN-LN:24-74 } \\
\text { tuô̂i }\end{array}$ \\
\hline$\leq 45$ tuối & 30 & 38,0 \\
\hline$>45$ tuối & 49 & 62,0 \\
\hline Giới tính: Nam & 27 & 34,2 \\
\hline Nũ̃ & 52 & 65,8 \\
\hline \multicolumn{3}{|l|}{ Nơi chốn } \\
\hline Tp.HCM & 63 & 79.8 \\
\hline Các tỉnh/thành khác & 16 & 20.2 \\
\hline \multicolumn{3}{|l|}{ Trình độ học vấn } \\
\hline$\leq$ Cấp 1 & 13 & 16.5 \\
\hline Cấp 2-Cấp 3 & 38 & 48.1 \\
\hline >Cấp 3 & 28 & 35.4 \\
\hline \multicolumn{3}{|c|}{ Thu nhập bình quân hàng tháng } \\
\hline$<5$ triệu đồng & 14 & 17.7 \\
\hline 5-10 triệu đông & 25 & 31,7 \\
\hline >10 triệu đônng & 4 & 50,6 \\
\hline $\begin{array}{l}\text { Thời gian mất răng } \\
\text { (tháng) }\end{array}$ & $\begin{array}{c}\text { TB } \pm Đ L C: \\
106,4 \pm 117,4\end{array}$ & $\begin{array}{c}\text { NN-LN: } \\
\text { 4-500 tháng }\end{array}$ \\
\hline $\begin{array}{l}\text { Thời gian mang hàm } \\
\text { giả (tháng) }\end{array}$ & $\begin{array}{c}\text { TB土ĐLC: } \\
88,5 \pm 95,5\end{array}$ & $\begin{array}{c}\text { NN-LN: } 0-480 \\
\text { tháng }\end{array}$ \\
\hline \multicolumn{3}{|c|}{ Mang hàm giả } \\
\hline
\end{tabular}

\begin{tabular}{|c|c|c|}
\hline $\begin{array}{c}\text { Không có hàm giả } \\
\text { cũ }\end{array}$ & 23 & 29,1 \\
\hline $\begin{array}{c}\text { Có hàm giả cũ và } \\
\text { không mang }\end{array}$ & 2 & 2,5 \\
\hline $\begin{array}{c}\text { Đang mang hàm } \\
\text { giả cũ }\end{array}$ & 54 & 68,4 \\
\hline
\end{tabular}

Đối tượng nghiên cứu có độ tuối trung bình là 47,5 tuổi, nữ chiếm $65,8 \%$. Đối tượng sống ở Tp. Hồ Chí Minh chiếm 79.8\%, thời gian mất răng trung bình là 106,4 tháng, thời gian mang hàm giả trung bình là 88,5 tháng và đối tượng đang mang hàm giả cũ chiếm $68,4 \%$, chưa từng mang hàm giả là $29,1 \%$.

Tác động của tình trạng mất răng lên chất lượng cuộc sống

Bảng 2. Phân bố giới tính, tuổi theo nhóm phuc hinh

\begin{tabular}{|c|c|c|c|c|}
\hline & $\begin{array}{c}\text { Cố định } \\
\text { n (\%) }\end{array}$ & $\begin{array}{c}\text { Hàm } \\
\text { khung } \\
\text { n (\%) }\end{array}$ & $\begin{array}{c}\text { Kết } \\
\text { hợp } \\
\text { n(\%) }\end{array}$ & p \\
\hline \multicolumn{5}{|c|}{ Giới tính } \\
\hline Nam & $15(55,6)$ & $12(44,4)$ & $0(0,0)$ & \multirow{2}{*}{ 12 $67^{*}$} \\
\hline Nữ & $31(59,6)$ & $19(36,5)$ & $2(3,9)$ & 0,67 \\
\hline
\end{tabular}

\begin{tabular}{|l|l|l|l|}
$\leq 45$ tuối & $27(90,0)$ & $3(10,0)$ & $0(0,0)$ \\
\hline
\end{tabular}

$>45$ tuối $19(38,8)|28(57,1)| 2(4,1) \mid<0,001 *$

*Kiểm định chính xác Fisher

Tỷ lệ nam làm phục hình cố định chiếm $55,6 \%$, nữ chiếm $59,6 \%$ phục hình cố định và $3,9 \%$ hàm kết hợp cả hai loại cố định và tháo lắp. Sự khác biệt không có ý nghĩa thống kê về các loại phục hình theo giới tính $(p>0,05)$.

ở nhóm tuổi, nhóm dưới 45 tuổi tî lệ làm phục hình cố định cầu răng chiếm $90 \%$, nhóm trên 45 tuổi tỉ lệ làm hàm khung tháo lắp chiếm $57,1 \%$. Sự khác biệt có ý nghĩa thống kê về các loại phục hình theo nhóm tuổi $(p<0,05)$.

Bảng 3. Điểm OHIP - 14 trước và sau điều trị 1 tháng $(n=79)$

\begin{tabular}{|c|c|c|c|c|c|}
\hline \multirow{2}{*}{ OHIP } & \multicolumn{2}{|c|}{ Trước điều trị } & \multicolumn{2}{|c|}{ Sau điêu trị 1 tháng } & p \\
\hline & TB \pm DLC & NN-LN & TB \pm ĐLC & NN-LN & \\
\hline Giới hạn chức năng & $2,1 \pm 2,0$ & $0-8$ & $0,9 \pm 1,1$ & $0-4$ & $<0,001^{* *}$ \\
\hline Đau thực thế & $3,4 \pm 1,8$ & $0-8$ & $2,1 \pm 1,4$ & $0-6$ & $<0,001^{*}$ \\
\hline Không thoải mái tâm lý & $3,0 \pm 2,1$ & $0-7$ & $1,5 \pm 1,3$ & $0-5$ & $<0,001^{* *}$ \\
\hline Thiếu năng thế chất & $3,0 \pm 1,8$ & $0-7$ & $1,8 \pm 1,3$ & $0-5$ & $<0,001^{*}$ \\
\hline Thiếu năng tâm lý & $2,5 \pm 1,8$ & $0-7$ & $1,3 \pm 1,3$ & $0-4$ & $<0,001^{* *}$ \\
\hline Thiếu năng xã hội & $1,8 \pm 1,4$ & $0-5$ & $1,0 \pm 1,1$ & $0-4$ & $<0,001^{* *}$ \\
\hline Tàn tật & $2,3 \pm 2,1$ & $0-10$ & $1,0 \pm 1,1$ & $0-4$ & $<0,001^{* *}$ \\
\hline Tống điếm OHIP 14 câu & $18,1 \pm 10,8$ & $0-40$ & $9,6 \pm 7,4$ & $0-29$ & $<0,001 * *$ \\
\hline
\end{tabular}

*Kiếm định ttest bắt cặp **Kiếm định Wilcoxon,

Sự khác biệt có ý nghĩa thống kê về điểm số trung bình OHIP-14 ở 7 lĩnh vực trước và sau điều trị phục hình 1 tháng $(\mathrm{p}<0,05)$.

Mối liên quan giữa điểm số OHIP-14 trước và sau điêu trị 1 tháng với các đặc điểm của mẫu nghiên cứu 
Bảng 4. Môi liên quan giữa điểm CLCS theo OHIP14 sau thời gian điều trị 1 tháng

\begin{tabular}{|c|c|c|}
\hline $\begin{array}{c}\text { Các yếu tố đăăc } \\
\text { điểm của mẫu } \\
\text { nghiên cứu }\end{array}$ & 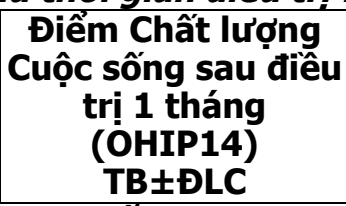 & $\mathbf{p}$ \\
\hline \multicolumn{3}{|c|}{ Tuối } \\
\hline$\leq 45$ tuối & $7,3 \pm 6,9$ & \multirow{2}{*}{$0,01 *$} \\
\hline$>45$ tuối & $11,1 \pm 7,4$ & \\
\hline \multicolumn{3}{|c|}{ Giới tính } \\
\hline Nam & $10,9 \pm 8,6$ & \multirow{2}{*}{$0,38^{*}$} \\
\hline Nữ & $8,9 \pm 6,7$ & \\
\hline \multicolumn{3}{|c|}{ Nơi chốn } \\
\hline Tp.HCM & $9,0 \pm 7,4$ & \multirow[b]{2}{*}{$0,15^{*}$} \\
\hline $\begin{array}{l}\text { Các tỉnh/thành } \\
\text { khác }\end{array}$ & $12,0 \pm 7,3$ & \\
\hline \multicolumn{3}{|c|}{ Trình độ học vấn } \\
\hline$\leq$ Cấp 1 & $11,5 \pm 9,7$ & \multirow{3}{*}{$0,13 * *$} \\
\hline Cấp 2-Cấp 3 & $8,9 \pm 6,9$ & \\
\hline >Cấp 3 & $8,4 \pm 6,0$ & \\
\hline \multicolumn{3}{|c|}{ Thu nhập bình quân hàng tháng } \\
\hline$<5$ triệu đồng & $10,2 \pm 7,2$ & \multirow{3}{*}{$0,91 * *$} \\
\hline 5-10 triệu đồng & $9,9 \pm 8,8$ & \\
\hline$>10$ triệu đồng & $9,2 \pm 6,6$ & \\
\hline \multicolumn{3}{|c|}{ Mang hàm giả } \\
\hline $\begin{array}{l}\text { Không có hàm } \\
\text { giả cũ }\end{array}$ & $9,2 \pm 7,1$ & \multirow{3}{*}{$0,13^{* *}$} \\
\hline \begin{tabular}{|c} 
Có hàm giả cũ và̀ \\
không mang
\end{tabular} & $21,0 \pm 2,8$ & \\
\hline \begin{tabular}{|c|} 
Đang mang hàm \\
giả cũ
\end{tabular} & $9,4 \pm 7,4$ & \\
\hline \multicolumn{3}{|l|}{ Loại phục hình } \\
\hline Cố định & $7,1 \pm 6,2$ & \multirow{3}{*}{$0,001 * *$} \\
\hline Hàm khung & $12,8 \pm 7,8$ & \\
\hline Kết hợp & $17,5 \pm 2,1$ & \\
\hline
\end{tabular}

*Kiểm định Mann Whitney,

**Kiểm định Kruskal-Wallis.

Sự khác biệt có ý nghĩa thống kê về điểm trung bình OHIP-14 sau điều trị 1 tháng theo nhóm tuổi và loại phục hình $(p<0,05)$. Không có sự khác biệt có ý nghĩa thống kê về điểm trung bình OHIP-14 theo giới tính, nơi chốn, trình độ học vấn, thu nhập bình quân hàng tháng và mang hàm giả $(p>0,05)$.

\section{BÀN LUẬN}

Đối tượng nghiên cứu có độ tuổi trung bình là 47,5 tuổi; trong đó người nhỏ nhất tham gia nghiên cứu là 24 tuổi và người lớn nhất là 74 tuổi. Tỉ lệ nữ trong nghiên cứu là $65,8 \%$, đối tượng sống ở Tp. Hồ Chí Minh chiếm 79,8\%, thời gian mất răng trung bình là 106,4 tháng với thời gian mất răng sớm nhất là 4 tháng và thời gian mất răng dài nhất là 500 tháng, thời gian mang hàm giả trung bình là 88,5 tháng với những người chưa từng mang hàm giả lần nào và thời gian mang hàm giả lâu nhất là 480 tháng.

Để đánh giá chất lượng cuộc sống chúng tôi sử dụng bảng câu hỏi OHIP-14 đáng tin cậy và được sử dụng rộng rãi ở nhiều quốc gia. Hơn nữa, bảng câu hỏi OHIP-14VN đã được Nguyễn Châu Thoa và CS [4] hiệu chỉnh phù hợp với ngôn ngữ Việt Nam và bảng câu hỏi được thực hiện trước và sau phục hình 1 tháng.

Có sự cải thiện về điểm số trung bình OHIP14 ở 7 lĩnh vực: giới han chức năng, đau thực thể, không thoải mái tâm lí, thiểu năng về thể chất, thiểu năng về tâm lí, thiểu năng về xã hội và tàn tât trước và sau điều trị phục hình 1 tháng. Điều này cho thấy điều trị phục hình răng có khả năng phục hồi chức năng răng miệng của đối tượng dẫn đến cải thiện đáng kể chất lượng cuộc sống hàng ngày của họ. Kết quả này phù hợp với nghiên cứu của Katerina Z và CS (2020) các đối tượng thuộc nhóm phục hình cố định, tháo lắp và loại kết hợp cả hai đều cải thiện chất lượng cuộc sống sau điều trị phục hình [2]. Nghiên cứu của Swelem và CS (2014) cho thây điểm OHIP-14 giảm đáng kể ở tất cả các nhóm điều trị ngoại trừ nhóm trẻ tuổi 30-40 tuổi được điêu trị bằng phục hình tháo lắp sau 6 tuần [8]. Trong nghiên cứu của Montero và CS (2013) thì phục hình tháo lắp hàm khung cải thiên đáng kể nhất, dễ vệ sinh hơn trong khi phục hình cố định câu răng có chức năng ăn uống tốt hơn và cả hai đều cải thiện chất lượng cuộc sống sau 1 tháng phuc hình [3]. Nghiên cứu của Amjid $\mathrm{N}$ và $\mathrm{CS}$ (2019) cho thấy đa số bệnh nhân hài lòng hơn ở 7 lĩnh vực của OHIP-14, tuy nhiên, hầu hết đối tượng cho rằng phục hình cố định sẽ dễ chịu hơn [1].

Nghiên cứu của chúng tôi có sự khác biệt về điểm số trung bình OHIP-14 có ý nghĩa thống kê với nhóm tuổi dưới và trên 45 tuổi, những đối tượng thuộc nhóm tuổi dưới 45 tuổi có điểm số

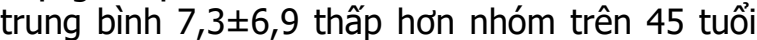
$11,1 \pm 7,4$, nghiên cứu của Reem $\mathrm{H}$ và CS (2018) [6] cho thây đối tượng nam có nhóm tuổi trẻ 3545 tuổi có điểm số trung bình OHIP thấp hơn, điều này có thể do vấn đề quan tâm đến chức năng, thẩm mỹ và khả năng thích nghi của người trẻ sẽ tốt hơn. Kết quả nghiên cứu cho thấy có sự khác biệt có ý nghĩa thống kê về điểm số trung bình OHIP-14 với các loại phục hình trong đó phục hình cố định cầu răng có điểm trung

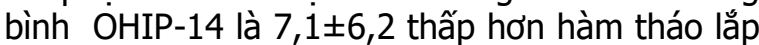
hàm khung có điểm trung bình OHIP-14 là $12,8 \pm 7,8$. Điều này phù hợp với nghiên cứu của Katerina Z và CS (2020) cho thấy phục hình cố 
định làm cải thiện chất lượng cuộc sống nhiêu hớn phục hình kết hợp và cuối cùng là phục hình tháo lắp. Nghiên cứu của Amjid N và CS (2019) cho thấy phục hình cố định câu răng giải quyết được các vấn đề thẩm mỹ và chức năng ăn nhai hơn phục hình tháo lắp [1].

\section{KẾT LUẦN}

Kết quả nghiên cứu cho thây thấy có sự cải thiện chất lượng cuộc sống sau điêuu trị phục hình cố định hoặc/và phục hình tháo lắp bán phân 1 tháng. Sự khác biệt có ý nghĩa thống kê vê điểm trung bình OHIP-14 sau điều trị 1 tháng theo nhóm tuổi và loại phục hình $(p<0,05)$.

\section{TÀI LIẸU THAM KHẢO}

1. Amjid N., Muhammad A. C., Babar A., Mariya K., Siddiq Y., Iqbal H., Irtifaq A. N. (2019), "Oral health- related quality of life in prosthodontics patients of Sardar Begum Dental College Peshawar", JKCD, 9(2), pp. 28-33.

2. Katerina Z., Cena' D., Natasa L., Ivona K., Sanja N. (2020), "The Impact of oral health on quality of

life in partially edentulous patients before and after prosthodontics rehabilitions", Journal of Hygienic Engineering and Design, pp.89-94.

3. Montero J., et al. (2013), "Self-perceived changes in oral health-related quality of life after receiving different types of conventional prosthetic treatments: a cohort follow-up study", J Dent, 41(6), pp. 493-503.

4. Nguyen T. C., et al.(2010), "Oral health status of adults in Southern Vietnam - a cross-sectional epidemiological study", BMC Oral Health, 10: p. 2.

5. Organization World Health (1997), WHOQOL Measuring quality of life. Programme on Mental Health. Division of Mental Health and Prevention of Substance Abuse, 13(2): p. 13.

6. Reem H. W., Elfatih I. E. (2018), "Impact of Removable Partial Denture on Quality-of-life of Sudanese Adults in Khartoum State", The Journal of Contemporary Dental Practice,19(1), pp.102-108.

7. Slade Gary D. (1997), "Measuring Oral Health and Quality of Life", Department of Dental Ecology, School of Dentistry, University of North Carolina,

8. Swelem A. A., et al.(2014), "Oral health-related quality of life in partially edentulous patients treated with removable, fixed, fixed-removable, and implant-supported prostheses", Int J Prosthodont, 27(4): p. 338-47.

\section{TỈ LỆ, ĐĂC ĐIỂM VÀ THÁI Độ XỬ TRÍ CHẤN THƯƠNG SỌ NÃO MÁU TỤ NGOÀI MÀNG CỨNG TẠI BỆNH VIÊ̂N ĐA KHOA TỈNH THÁI BÌNH}

\section{TÓM TẮT}

Mục tiêu: Nhận xét tỉ lệ, nguyên nhân, đặc điểm máu tư ngoài màng cứng do chấn thương so não điều trị tại Bệnh viện Đa khoa tỉnh Thái Bình. Phương pháp: Mô tả cắt ngang 75 bệnh nhân máu tụ ngoài màng cứng do chấn thương sọ não điều trị tại Bệnh viên Đa khoa tỉnh Thái Bình trong khoảng thời gian từ tháng 2 tới tháng 9 năm 2020. Kết quả: Tỉ lệ máu tụ ngoài cứng $(14,0 \%)$ : 75/534 bệnh nhân chấn thương sọ não nhập viện điều trị trong khoảng thời gian 8 tháng. 75 bênh nhân gồm 64 nam $(85,3 \%) ; 11$ nữ $(14,7 \%)$; Tuổi trung bình: 34,2 \pm 21,2 tuổi; Độ tuổi lao động chiếm nhiêu nhất $47 / 75$ chiếm $(62,6 \%)$. Nguyên nhân do tai nạn giao thông chiếm cao nhất (68\%); Lâm sàng $74 / 75$ bênh nhân mức độ nhẹ $(98,7 \%)$; Cắt lớp vi tính: Vị trí máu tu vùng thái dương $(70,7 \%)$; vị trí trán $(36,0 \%)$; vùng đỉnh $(14,7 \%)$. Xử trí: điều trị nội $84,0 \%$; phẫu thuật lấy máu tụ 16,0\%. Kết quả ra viện tốt $98,7 \%$; xấu 1 bệnh nhân; không có tử vong. Kết luận: Tí lệ máu tụ ngoài

*Trường Đại học Y Dược Thái Bình Chiu trách nhiếm chính: Vũ Minh Hải Email: vuminhhai777@gmail.com Ngày nhận bài: 19.3.2021

Ngày phản biện khoa học: 14.5.2021

Ngày duyệt bài: 21.5.2021
Vũ Minh Hải*

màng cứng chiếm $(14,0 \%)$ trong tổng số bệnh nhân chấn thương sọ não. Nguyên nhân do tai nạn giao thông gặp nhiều nhất (68\%). Nam giới chiếm đa số $(85,3 \%)$. Mức độ lâm sàng nhẹ tương ứng với thể tích máu tụ nội sọ nhỏ, nên xử trí đa số là điêu trị nội khoa. so não.

Tứ khóa: Máu tụ ngoài màng cứng; chấn thương

\section{SUMMARY}

\section{RATE, CHARACTERISTICS AND ATTITUDE OF MANAGING TRAUMATIC BRAIN INJURY WITH EPIDURAL HEMATOMA AT \\ THAI BINH GENERAL HOSPITAL}

Objectives: To evaluate the rate, causes, and characteristics of epidural hematoma due to traumatic brain injury treated at Thai Binh General Hospital. Methods: Cross-sectional descriptive study in 75 patients with epidural hematoma due to traumatic brain injury treated at Thai Binh General Hospital between February and September 2020. Results: Percentage of hematoma (14.0\%): 75/534 traumatic brain injury patients hospitalized for a period of 8 months. 75 patients, including 64 males $(85.3 \%) ; 11$ females $(14.7 \%)$; Median age: $34.2 \pm 21.2$ years old; Working age accounted for the most 47/75 (62.6\%). Traffic accidents composed the highest $(68 \%)$ in causes; Clinically: 74/75 patients with minor level $(98.7 \%) ;$ CT scan: temporal epidural hematoma 